\title{
Impact of G-CSF on expressions of Egr-1 and VEGF in acute ischemic cerebral injury
}

\author{
DIAN-GUI ZHOU ${ }^{1}$, YANG-HONG SHI ${ }^{2}$ and YOU-QIANG CUI ${ }^{3}$ \\ ${ }^{1}$ Department of Neurology, Zhongshan Torch Development Zone Hospital, Zhongshan, Guangdong 528437; \\ ${ }^{2}$ Department II of Neurology, The First Hospital of Yulin, Yulin, Shaanxi 719000; ${ }^{3}$ Department of Neurosurgery, \\ Qianfoshan Hospital Affiliated to Shandong University, Jinan, Shandong 250014, P.R. China
}

Received December 26, 2017; Accepted June 25, 2018

DOI: $10.3892 /$ etm.2018.6486

\begin{abstract}
The aim of the present study was to investigate the protective effect of granulocyte colony-stimulating factor (G-CSF) on acute ischemic cerebral injury, and its mechanism through the impact of G-CSF on early growth response-1 (Egr-1) and vascular endothelial growth factor (VEGF) expressions. Male Sprague-Dawley (SD) rats were divided them into three groups, i.e., the sham, model and G-CSF groups to measure the effect of G-CSF on the volume of cerebral infarction and level of lactate dehydrogenase (LDH) in rats. Hematoxylin and eosin (H\&E) staining method was performed for histopathological examination. Reverse transcription-polymerase chain reaction (RT-PCR) and western blot analysis were used to detect the mRNA and protein expressions of Egr-1 and VEGF in different groups. Furthermore, Statistical Product and Service Solutions (SPSS) 17.0 software was applied to detect the differences in the expression of Egr-1 and VEGF between the two groups. Compared with the sham group, we found that the volume of cerebral infarction and LDH content in the model group were significantly elevated. By contrast, in the model group, those indicators in the G-CSF group were obviously decreased. H\&E staining results also showed that G-CSF could decrease the necrotic area in cerebral infarction and the incidence of inflammation, and sustain the integrity of the molecular structure. Immunofluorescence staining results revealed that the protein expressions of Egr-1 and VEGF in the model group were all significantly decreased, while those in the G-CSF group were remarkably elevated. RT-PCR and western blot analysis revealed that the mRNA and protein expressions of Egr-1 and VEGF in the model group were decreased obviously, but those in the G-CSF group were elevated significantly, and the differences between the two groups showed statistical significance $(\mathrm{P}<0.05)$. G-CSF
\end{abstract}

Correspondence to: Dr You-Qiang Cui, Department of Neurosurgery, Qianfoshan Hospital Affiliated to Shandong University, 16766 Jingshi Road, Jinan, Shandong 250014, P.R. China

E-mail: caotan352@163.com

Key words: G-CSF, acute ischemic cerebral injury, Egr-1, VEGF manifests a significant protective effect on the acute ischemic cerebral injury, which may be realized through its effect on the expressions of Egr-1 and VEGF.

\section{Introduction}

In recent years, acute ischemic cerebral injury has become a kind of disease with an extremely high incidence rate and poor prognosis, and for some severe conditions, it can even be life-threatening (1). Generally, it is originated from the cerebral thrombosis and embolism, and, after onset of ischemia, timely recovery of blood supply is critical to the reduction of apoptosis in neurons and repair of neurological functions. Ischemic angiogenesis is closely related to the progression and outcome of cerebrovascular diseases. However, current treatment method for acute ischemic cerebral injury requires further research and development (2-4).

Granulocyte colony-stimulating factor (G-CSF), as a member of hematopoietic growth factor family, can promote the proliferation, differentiation and survival of hematopoietic stem cells with the obvious protective effect on neurons in peripheral and central nerve system (5). In the present study, we aimed to establish the models of acute ischemic cerebral injury in rats to investigate the changes in early growth response-1 (Egr-1) and vascular endothelial growth factor (VEGF) in cerebral tissues after intervention with G-CSF, thereby exploring the potential mechanism of G-CSF in protecting nerves, and providing new pathways for clinical treatment of acute ischemic cerebral injury.

\section{Materials and methods}

\section{Materials}

Experiment animals and grouping. After Sprague-Dawley (SD) rats (180-220 g) were acclimatized to the environment, they were randomly divided into the sham group $(n=10)$, the model group $(\mathrm{n}=10)$ and the G-CSF group $(100 \mu \mathrm{g} / \mathrm{kg}, \mathrm{n}=10)$. G-CSF was given through neck subcutaneous injection once per day for 7 consecutive days. Subsequently, access of food to rats was forbidden for $12 \mathrm{~h}$ but they had free access to water. The rats were kept in cage with controlled temperature and light cycles ( $24^{\circ} \mathrm{C}$ and $12 / 12$ light cycles). Longa's method was applied to establish the middle cerebral artery occlusion models 
in rats. The humidity was $60 \pm 10 \%$. The study was approved by the Ethics Committee of Zhongshan Torch Development Zone Hospital (Zhongshan, China).

Major reagents. TRIzol extraction kit for total RNA (Tiangen, Co., Ltd., Beijing, China); real-time polymerase chain reaction (RT-PCR) kit for reverse transcription (Tiangen); bicinchoninic acid (BCA) kit for protein quantification (Beyotime Institute of Biotechnology, Shanghai, China); extraction kit for total protein (Nanjing KeyGen Biotech, Co., Ltd., Nanjing, China); immunopreticipation (IP) lysis kit (Beyotime Institute of Biotechnology); lactate dehydrogenase (LDH) kit (Nanjing Jiancheng Biotech Co., Ltd., Nanjing, China); primary antiglyceraldehyde-phosphate dehydrogenase (GAPDH), Egr-1 monoclonal antibodies and the relevant secondary antibodies (Cell Signaling Technology, Boston, MA, USA) and VEGF monoclonal antibody (ProteinTech Group, Inc., Wuhan, China) were used in the present study.

\section{Experiment methods}

Measurement of the volume of cerebral infarction and relevant indicators. After rats in each group were anesthetized, they were immediately sacrificed and brain tissue was removed. Samples were then stained for $30 \mathrm{~min}$ at $37^{\circ} \mathrm{C}$ in the dark in $1 \%$ triphenyltetrazolium chloride (TTC), and then fixed in $10 \%$ formaldehyde. The infarction area in each layer was then calculated, and LDH content in brain tissues in the three groups was assayed in accordance with the LDH kit.

Histopathological examination. Brain tissues in each group were embedded in paraffin to prepare the paraffin samples which were later sliced into sections of $5 \mu \mathrm{m}$. After hematoxylin and eosin (H\&E) staining, the sections were placed under a microscope (x100) (Roche Diagnostics, Basel, Switzerland) for histopathological observation.

Immunofluorescence analysis. Paraffin sections of brain tissues were collected from each group, and, after dewaxing with xylene and dehydration using alcohol of gradient concentration, the antigen retrieval was carried out. Sections were then rinsed with $0.01 \mathrm{M}$ phosphate-buffered saline (PBS, pH 7.4) 3 times (5 min/time), and blocked in a wet box containing $10 \%$ bull serum albumin (BSA) for $30 \mathrm{~min}$. Subsequently, on sections, fluorescence-labeled antibodies that were diluted appropriately (1:70) were placed in the wet box and the sections were then incubated at $4^{\circ} \mathrm{C}$ overnight. After sections were rinsed with PBS (pH 7.4) 3 times, fluorescent secondary antibodies (1:100) were added onto the sections in the dark, and placed in the wet box for incubation for $2 \mathrm{~h}$ at $37^{\circ} \mathrm{C}$. The sections were then observed and photographed under the fluorescent microscopes (Olympus Corporation, Tokyo, Japan).

RT-PCR analysis. Brain tissues that were collected from each group were rapidly transferred to the Eppendorf (EP) tubes containing RNAiso Plus extraction solution and placed at room temperature for $5 \mathrm{~min}$ for sufficient lysis. After centrifugation at $12,000 \mathrm{x} \mathrm{g}$ and $4^{\circ} \mathrm{C}$ for $5 \mathrm{~min}$, the supernatant was well mixed with $0.2 \mathrm{ml}$ chloroform and placed at room temperature for $5 \mathrm{~min}$. The solution was centrifuged again at
Table I. Primer sequences of relevant genes in RT-PCR analysis.

\begin{tabular}{ll}
\hline Gene name & \multicolumn{1}{c}{ Primer sequence } \\
\hline Egr-1 & 5'-3' TCGGCTCCTTTCCTCACTCA \\
VEGF & 3'-5' CTCATAGGGTTGTTCGCTCGG \\
& 5'-3' ATGGCAGAAGGAGGAGGG \\
$\beta$-actin & 3'-5' CGAAACGCTGAGGGAGGCT \\
& 5'-3' GAGCCGGGAAATCGTGCGT \\
& 3'-5' GGAAGGAAGGCTGGAAGATG \\
\hline
\end{tabular}

$12,000 \mathrm{x} \mathrm{g}$ and $4^{\circ} \mathrm{C}$ for $15 \mathrm{~min}$, and, isopropanol of the same volume was added and well mixed to the supernatant. After being placed at room temperature for $10 \mathrm{~min}$, it was centrifuged at $12,000 \mathrm{x} \mathrm{g}$ and $4^{\circ} \mathrm{C}$ for $10 \mathrm{~min}$, and sediment was preserved while the supernatant was discarded. In the sediment, $1 \mathrm{ml} \mathrm{75 \%} \mathrm{alcohol} \mathrm{was} \mathrm{added} \mathrm{and} \mathrm{mixed} \mathrm{well} \mathrm{followed}$ by centrifugation at $12,000 \mathrm{x} \mathrm{g}$ and $4^{\circ} \mathrm{C}$ for $5 \mathrm{~min}$, which was repeated once after the supernatant was then removed. After RNA sediment was fully removed through rinsing, the liquid was replaced with RNase-free water. Some of the total RNA solution was extracted and diluted with RNase-free water to a concentration of $1 \mu \mathrm{g} / \mu \mathrm{l}$. In accordance with the requirement of the PrimeScript ${ }^{\circledR}$ RT reagent kit with gDNA Eraser, reaction solution for reverse transcription was prepared, and cDNA was obtained through reverse transcription with the RNA samples and preserved at $-20^{\circ} \mathrm{C}$. Thereafter, according to the instruction of SYBR ${ }^{\circledR}$ Premix Ex Taq ${ }^{\mathrm{TM}}$ II (Tli RNaseH Plus), we detected the level of mRNA expression. The relevant RNA primer sequences used in this procedure are shown in Table I.

Western blotting assay. Brain tissues collected from all the groups were rinsed with icy normal saline followed by measurement of protein concentration with BCA kit and preservation at $-80^{\circ} \mathrm{C}$ for later use. According to the instructions of total protein extraction kit, the IP lysis buffer supplemented with phenylmethanesulfonyl fluoride (PMSF) and protease inhibitor was added in the tissues which were later ground on ice. Tissue samples were then centrifuged at $4^{\circ} \mathrm{C}$ and $12,000 \mathrm{xg}$ for $10 \mathrm{~min}$ of homogenization, and the supernatant was taken for centrifugation at $4^{\circ} \mathrm{C}$ and $12,000 \mathrm{x} \mathrm{g}$ for $20 \mathrm{~min}$. With the supernatant, protein samples containing the same content of total protein, after protein quantification according to the relevant kit instruction, were loaded in the sampling holes for electrophoresis under a constant voltage of $220 \mathrm{~V}$. Electrophoresis was stopped until the bromophenol blue reached the bottom of gel. Based on the molecular weight of targeting proteins, gel was trimmed and placed in the transfer buffer. Polyvinylidene fluoride (PVDF) membrane was also trimmed in accordance with the size of the gel, and then soaked in methanol for $10 \mathrm{sec}$. The PVDF membrane and filter paper were placed in the transfer buffer, and later, those materials were all placed in the transfer machine in the following sequence: positive electrode, filter paper, PVDF membrane, gel, filter paper and negative electrode. Membrane transfer was then carried out under a constant voltage of $110 \mathrm{~V}$ for appro- 

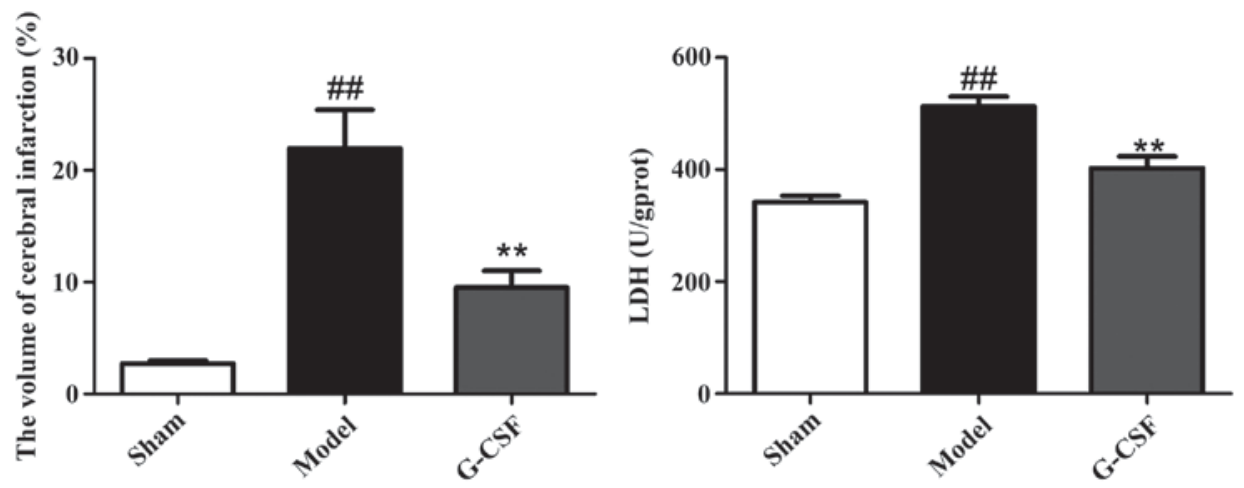

Figure 1. Effect of G-CSF on the volume of cerebral infarction and LDH content in brain tissues in rats. In comparison with the sham group, the volume of cerebral infarction in rats of the model group is significantly enlarged $\left({ }^{\# \#} \mathrm{P}<0.01\right)$. By contrast, compared with the model group, a significant decrease is identified in the volume of cerebral infarction in rats of the G-CSF group $\left.{ }^{* * *} \mathrm{P}<0.01\right)$.
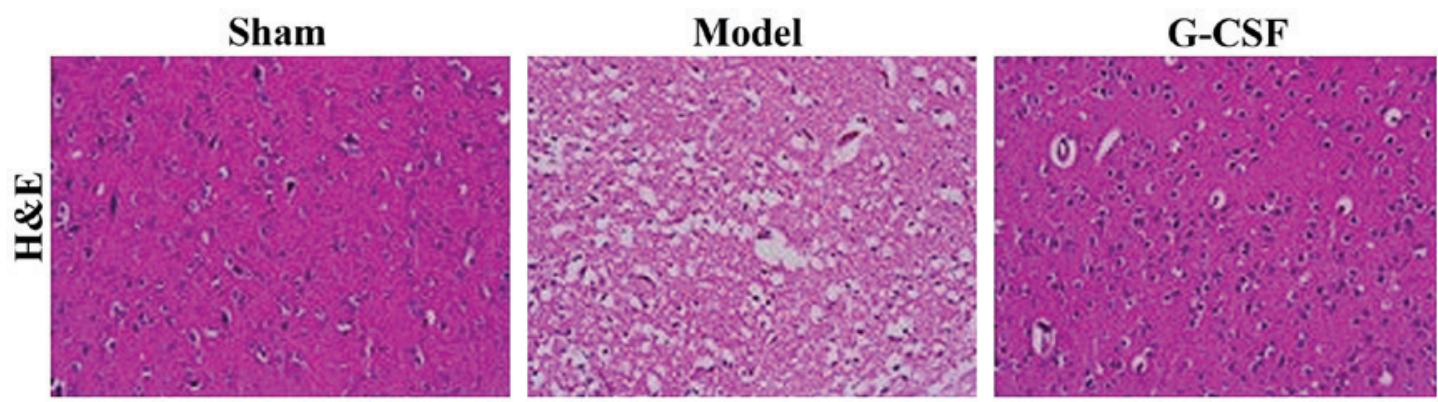

Figure 2. H\&E staining results of the effect of G-CSF on MCAO rats (magnification, x100).

priate time until the protein on the gel was transferred on the PVDF membrane. Thereafter, PVDF membrane with protein was blocked in $5 \%$ skimmed milk on a shaker for $3 \mathrm{~h}$, and the rabbit anti-rat Egr-1, and GAPDH monoclonal antibodies (1:1,000; cat. nos. 4154 and 2118), rabbit anti-rat VEGF monoclonal antibody (1:1,000; cat. no. 19003-1-AP) was added on the membrane for incubation at $4^{\circ} \mathrm{C}$ overnight. The next day, membrane was sufficiently washed by Tween-20 + Tris-buffer saline (TTBS) 3 times (10 min/time), and then incubated with goat anti-rabbit secondary polyclonal antibody $(1: 2,000$; cat. no. 7074) for $1 \mathrm{~h}$ at room temperature. Enhanced chemiluminescence (ECL) reagent was added for color development and photographing after the membrane was washed with TTBS 3 times (10 min/time).

Statistical analysis. Statistical Product and Service Solutions (SPSS) 17.0 software (SPSS, Inc., Chicago, IL, USA) was applied in statistical analysis, and experiment data were presented as mean \pm standard deviation (SD). Data analysis was carried out through analysis of variance and the post hoc test was LSD test for multiple comparisons or t-test for intergroup comparisons. $\mathrm{P}<0.05$ suggested that the difference had statistical significance.

\section{Results}

Effect of G-CSF on the volume of cerebral infarction and LDH content in brain tissues in rats. As shown in Fig. 1 in comparison with the sham group, the volume of cerebral infarction in rats of the model group was significantly enlarged.
By contrast, compared with the model group, a significant decrease was identified in the volume of cerebral infarction in rats of the G-CSF group. In addition, G-CSF could reduce the level of LDH in brain tissues significantly.

Effect of G-CSF on the histopathological examination of rats with acute ischemic cerebral injury. The H\&E staining results of histopathological examination of brain tissues are shown in Fig. 2. In the sham group, the brain structure of rats appeared to be integral, while rats in the model group manifested massive necrotic cells, and destructed molecular structures. Compared with the model group, we found that the necrotic area was reduced in the G-CSF group, suggesting the cerebral injury was ameliorated.

Immunofluorescent staining results of Egr-1 and VEGF. The immunofluorescence analysis revealed that compared with the sham group, the protein expressions of Egr-1 and VEGF in brain tissues of rats in the model group were reduced significantly, while those in the G-CSF group were increased evidently $(\mathrm{P}<0.01)$ (Fig. 3).

$R T-P C R$ results of the effect of $G-C S F$ on $m R N A$ expression of Egr-1 and VEGF in MCAO rats. As shown in Fig. 4, the RT-PCR results revealed that compared with the sham group, the mRNA expressions of Egr-1 and VEGF in brain tissues of rats in the model group were reduced significantly. By contrast, in comparison with the model group, the mRNA expression of Egr-1 and VEGF was increased significantly in brain tissues of rats in the G-CSF group. 


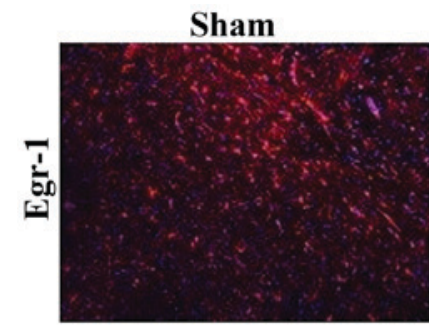

Sham

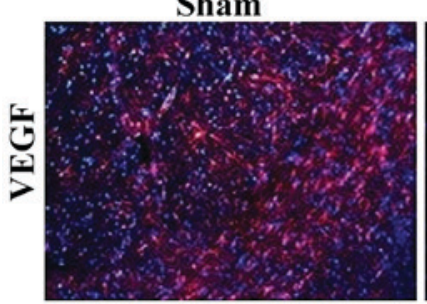

Model

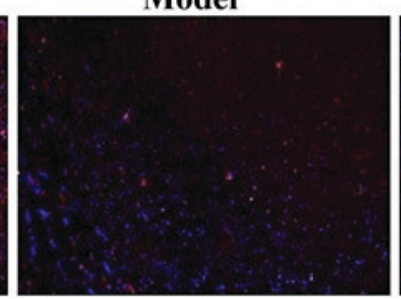

Model

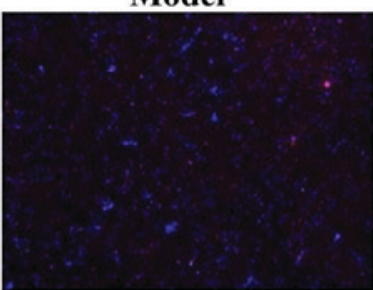

G-CSF

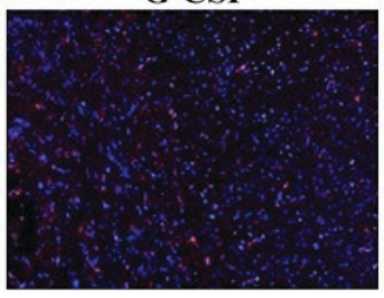

G-CSF

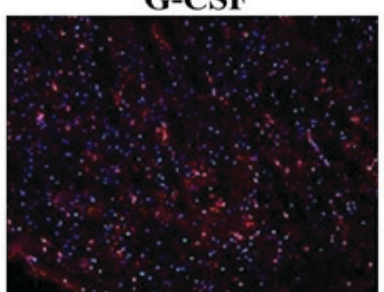

Figure 3. Immunofluorescence staining results of the effect of G-CSF on the expressions of Egr-1 and VEGF of MCAO rats (magnification, x100).
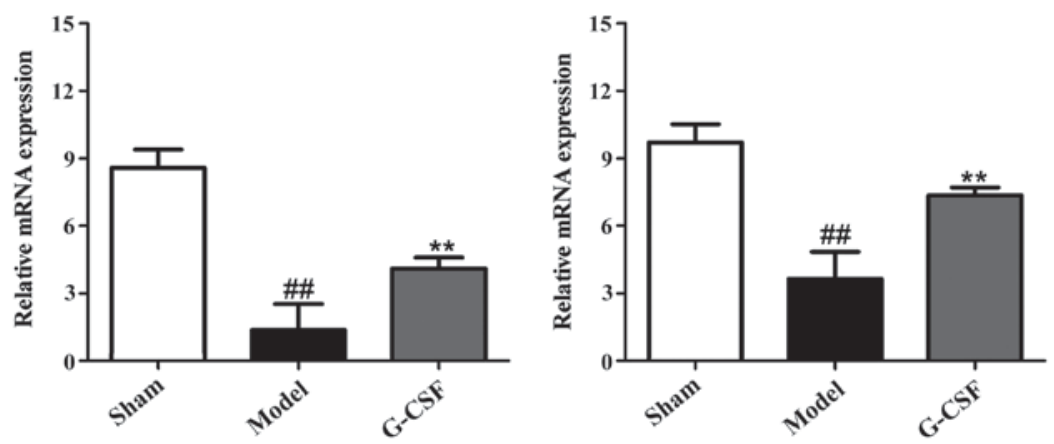

Figure 4. RT-PCR results of the effect of G-CSF on mRNA expression of Egr-1 and VEGF in MCAO rats. Compared with the sham group, the mRNA expression of Egr-1 and VEGF in brain tissues of rats in the model group are significantly reduced $\left({ }^{\#} \mathrm{P}<0.01\right)$. Compared with the model group, the mRNA expression of Egr-1 and VEGF are increased significantly in brain tissues of rats in the G-CSF group $\left({ }^{* *} \mathrm{P}<0.01\right)$.
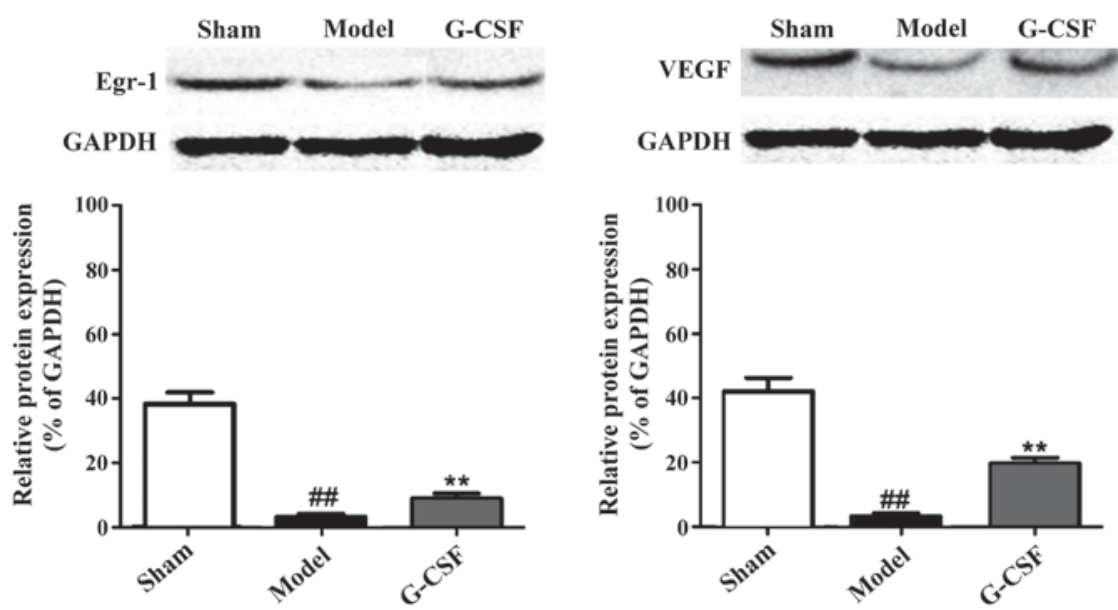

Figure 5. Western blot analysis results of the effect of G-CSF on the protein expression of Egr-1 and VEGF in MCAO rats. In comparison with the sham group, the protein expression of Egr-1 and VEGF in the brain tissues of rats in the model group are significantly reduced (\#\# $\mathrm{P}<0.01)$. Compared with the model group, the protein expression of Egr-1 and VEGF in brain tissues of rats in the G-CSF group are significantly elevated $\left({ }^{* *} \mathrm{P}<0.01\right)$.

Western blot analysis results of the effect of $G$-CSF on the protein expression of Egr-1 and VEGF in MCAO rats. In Fig. 5, the western blot analysis revealed that in comparison with the sham group, protein expression of Egr-1 and VEGF in the brain tissues of rats in the model group was significantly reduced. Compared with the model group, the protein expression of Egr-1 and VEGF in brain tissues of rats in the G-CSF group was significantly elevated. This result confirmed that G-CSF can ameliorate the acute ischemic cerebral injury by regulating the expressions of Egr-1 and VEGF. 


\section{Discussion}

Acute ischemic cerebral injury has gradually become a global killer with characteristics including high incidence rate, high mortality rate and high morbidity rate $(6,7)$. Cerebral ischemia can damage the brain cells, and once the blood perfusion is recovered, ischemic injury is further aggravated, known as cerebral ischemia-reperfusion injury (8-10). Pathogenesis leading to acute ischemic cerebral injury remains elusive, which makes the search for effective, reliable and feasible treatment methods for this disease more urgent. In recent years, G-CSF has gained more and more attention of researchers for its special effect (11).

G-CSF, as a kind of cytokine in hematopoietic stem cells, can promote the survival, proliferation and maturity of neutrophilic cell lines. G-CSF exists mostly in the bone marrow stem cell of patients (12-14). Previous findings have shown that G-CSF has a promising protective effect on nerves, and it can promote angiogenesis and exert an anti-inflammatory effect by regulating varying molecular mechanisms, such as mobilizing the generation of hematopoietic stem cells, inhibiting their apoptosis, facilitating the differentiation of neurons and antagonizing the inflammatory responses (15-18). Previous findings have shown that Egr-1 and VEGF have key roles in the regulation of growth and development of vessels, and are also critical to ischemic diseases. Additionally, they can facilitate the migration of hematopoietic stem cells with protective effects on nerves $(19,20)$.

In this study, male SD rats used as subjects, were divided the sham, model and G-CSF groups to measure the effect of G-CSF on the volume of cerebral infarction and level of LDH in rats. The H\&E staining method was performed for histopathological examination. Through RT-PCR and western blot analysis, we detected the mRNA and protein expressions of Egr-1 and VEGF in different groups. Furthermore, SPSS 17.0 software was applied to detect the differences in expression of Egr-1 and VEGF between the two groups. Results showed that compared with the sham group, the volume of cerebral infarction and LDH content in the model group were significantly elevated, whereas in the model group, those indicators in the G-CSF group were obviously decreased. H\&E staining results also showed that G-CSF could decrease the necrotic area in cerebral infarction and the incidence of inflammation, and sustain the integrity of molecular structure. Immunofluorescence staining results revealed that the protein expression of Egr-1 and VEGF in the model group were all significantly decreased, while those in the G-CSF group were significantly elevated. RT-PCR and western blot analysis revealed that the mRNA and protein expression of Egr-1 and VEGF in the model group was decreased obviously, but those in the G-CSF group were elevated significantly.

Taken together, G-CSF manifests a significant protective effect on acute ischemic cerebral injury, which is generated through its impact on the expression of Egr-1 and VEGF. The results of this study are expected to provide new therapeutic procedures for treatment of acute ischemic cerebral injury, and other effects of G-CSF on acute ischemic cerebral injury require more in-depth studies.

\section{Acknowledgements}

Not applicable.

\section{Funding}

No funding was received.

\section{Availability of data and materials}

The datasets used and/or analyzed during the current study are available from the corresponding author on reasonable request.

\section{Authors' contributions}

DGZ measured the volume of cerebral infarction and relevant indicators. YHS helped with histopathological examination. DGZ and YQC were reponsible for immunofluorescence, analysis and PCR. All authors read and approved the final manuscript.

\section{Ethics approval and consent to participate}

The study was approved by the Ethics Committee of Zhongshan Torch Development Zone Hospital (Zhongshan, China).

\section{Patient consent for publication}

Not applicable.

\section{Competing interests}

The authors declare that they have no competing interests.

\section{References}

1. Du H, Naqvi H and Taylor HS: Ischemia/reperfusion injury promotes and granulocyte-colony stimulating factor inhibits migration of bone marrow-derived stem cells to endometrium. Stem Cells Dev 21: 3324-3331, 2012.

2. Fan L, Chen L, Chen X and Fu F: A meta-analysis of stem cell mobilization by granulocyte colony-stimulating factor in the treatment of acute myocardial infarction. Cardiovasc Drugs Ther 22: 45-54, 2008.

3. Dai W, Hale SL, Martin BJ, Kuang JQ, Dow JS, Wold LE and Kloner RA: Allogeneic mesenchymal stem cell transplantation in postinfarcted rat myocardium: Short- and long-term effects. Circulation 112: 214-223, 2005.

4. Fuchs S, Baffour R, Zhou YF, Shou M, Pierre A, Tio FO, Weissman NJ,Leon MB, Epstein SE and Kornowski R: Transendocardial delivery of autologous bone marrow enhances collateral perfusion and regional function in pigs with chronic experimental myocardial ischemia. J Am Coll Cardiol 37: 1726-1732, 2001.

5. Schneider A, Krüger C, Steigleder T, Weber D, Pitzer C, Laage R, Aronowski J, Maurer MH, Gassler N, Mier W, et al: The hematopoietic factor G-CSF is a neuronal ligand that counteracts programmed cell death and drives neurogenesis. J Clin Invest 115: 2083-2098, 2005.

6. Schäbitz WR, Kollmar R, Schwaninger M, Juettler E, Bardutzky J, Schölzke MN, Sommer C and Schwab S: Neuroprotective effect of granulocyte colony-stimulating factor after focal cerebral ischemia. Stroke 34: 745-751, 2003.

7. Kawamoto A, Gwon HC, Iwaguro H, Yamaguchi JI, Uchida S, Masuda H, Silver M, Ma H, Kearney M, Isner JM, et al: Therapeutic potential of ex vivo expanded endothelial progenitor cells for myocardial ischemia. Circulation 103: 634-637, 2001.

8. Murry CE, Soonpaa MH, Reinecke H, Nakajima H, Nakajima HO, Rubart M,Pasumarthi KBS, Virag JI, Bartelmez SH, Poppa V, et al: Haematopoietic stem cells do not transdifferentiate into cardiac myocytes in myocardial infarcts. Nature 428: 664-668, 2004.

9. Olivetti G, Capasso JM, Meggs LG, Sonnenblick EH and Anversa P: Cellular basis of chronic ventricular remodeling after myocardial infarction in rats. Circ Res 68: 856-869, 1991. 
10. Park KI, Hack MA, Ourednik J, Yandava B, Flax JD, Stieg PE, Gullans S, Jensen FE, Sidman RL, Ourednik V, et al: Acute injury directs the migration, proliferation, and differentiation of solid organ stem cells: Evidence from the effect of hypoxia-ischemia in the CNS on clonal 'reporter' neural stem cells. Exp Neurol 199: 156-178, 2006

11. Pastuszko P1, Liu H, Mendoza-Paredes A, Schultz SE, Markowitz SD, Greeley WJ, Wilson DF and Pastuszko A: Regulatory pathways to neuronal injury or survival are dependent on the rate of low flow cardiopulmonary bypass following circulatory arrest in newborn piglets. Eur J Cardiothorac Surg 31: 899-905, 2007.

12. Rosenstrauch D, Poglajen G, Zidar N and Gregoric ID: Stem celltherapy for ischemic heart failure. Tex Heart Inst J 32: 339-347, 2005.

13. Tang YL, Zhao Q, Qin X, Shen L, Cheng L, Ge J and Phillips MI: Paracrine action enhances the effects of autologous mesenchymal stem cell transplantation on vascular regeneration in rat model of myocardial infarction. Ann Thorac Surg 80: 229-236, discussion 236-237, 2005

14. Xiao BG, Lu CZ and Link H: Cell biology and clinical promise of G-CSF: Immunomodulation and neuroprotection. J Cell Mol Med 11: 1272-1290, 2007.

15. Pastuszko P, Schears GJ, Pirzadeh A, Greeley WJ, Wilson DF and Pastuszko A: Effect of granulocyte colony stimulating factor (G-CSF) on expression of select proteins involved in apoptosis in a neonatal piglet brain following cardiopulmonary bypass (CPB) and deep hypothermic circulatory arrest (DHCA). J Thorac Cardiovasc Surg 143: 1436-1442, 2012.
16. Gibson CL, Bath PM and Murphy SP: G-CSF reduces infarct volume and improves functional outcome after transient focal cerebral ischemia in mice. J Cereb Blood Flow Metab 25: 431-439, 2005.

17. Lee ST, Chu K, Jung KH, Ko SY, Kim EH, Sinn DI, Lee YS, Lo EH, Kim M and Roh JK: Granulocyte colony-stimulating factor enhances angiogenesis after focal cerebral ischemia. Brain Res 1058: 120-128, 2005

18. Tang YL, Zhao Q, Zhang YC, Cheng L, Liu M, Shi J, Yang YZ, Pan C, Ge J and Phillips MI: Autologous mesenchymal stem cell transplantation induce VEGF and neovascularization in ischemic myocardium. Regul Pept 117: 3-10, 2004.

19. Cheng Z, Liu X, Ou L, Zhou X, Liu Y, Jia X, Zhang J, Li Y and Kong D: Mobilization of mesenchymal stem cells by granulocyte colony-stimulating factor in rats with acute myocardial infarction. Cardiovasc Drugs Ther 22: 363-371, 2008.

20. Su H, Lu R and Kan YW: Adeno-associated viral vector-mediated vascular endothelial growth factor gene transfer induces neovascular formation in ischemic heart. Proc Natl Acad Sci USA 97: 13801-13806, 2000.

This work is licensed under a Creative Commons Attribution-NonCommercial-NoDerivatives 4.0 International (CC BY-NC-ND 4.0) License. 